\title{
Epidemiology of Goiters in Sri Lanka with Geographic Information System Mapping: Population-based Cross-sectional Study
}

\author{
${ }^{1}$ Pramodh Chandrasinghe, ${ }^{2}$ Ranil Fernando, ${ }^{3}$ Sumal Nandasena, ${ }^{4}$ Arunasalam Pathmeswaran
}

\begin{abstract}
Background: Sri Lanka is considered an endemic region for goiter. Early researchers have suggested the existence of a goiter belt based on rainfall pattern. Subsequent studies done in school children have challenged this theory. Current study is a community-based cross-sectional study done to assess the epidemiology of goiters. Geographic information system (GIS) mapping of the goiter prevalence is developed for the first time in Sri Lanka.
\end{abstract}

Methods: Study subjects were selected using a multi-staged cluster sampling with probability proportionate to size (PPS) method. Examination for the presence of goiters was done by two trained investigators and graded. ArcGIS 10 software was used for geostatistical analysis and developing a map by interpolating the data collected for the first time in Sri Lanka.

Results: A total of 5200 individuals (female-66\%, median age -38 years; range 10-92 years) were assessed and 426 goiters were detected. The adjusted prevalence rate of goiters was $6.8 \%$. Kriging interpolation method was used to develop the most appropriate epidemiological map clustering of high prevalence areas with scattered pockets of high prevalence was observed. Current map does not show a prevalence pattern in relation to the rainfall or elevation above sea level as proposed in the past.

Conclusion: Goiter prevalence in Sri Lanka appears to be different from earlier proposed patterns. Use of GIS has contributed to develop a comprehensive epidemiological map of goiters demonstrating the absence of an endemic goiter belt in the wet zone as proposed earlier.

Keywords: Epidemiology, GIS mapping, Goiter prevalence.

How to cite this article: Chandrasinghe $P$, Fernando $R$, Nandasena S, Pathmeswaran A. Epidemiology of Goiters in Sri Lanka with Geographic Information System Mapping:

\footnotetext{
${ }^{1}$ Lecturer, ${ }^{2,4}$ Professor, ${ }^{3}$ Researcher

1,2Department of Surgery, Faculty of Medicine, University of Kelaniya, Ragama, Sri Lanka

${ }^{3}$ Ministry of Healthcare and Nutrition, Center for Collaborative Health Research, National Institute of Health Science Sri Lanka, India

${ }^{4}$ Department of Public Health, Faculty of Medicine, University of Kelaniya, Ragama, Sri Lanka
}

Corresponding Author: Pramodh Chandrasinghe, Lecturer Department of Surgery, Faculty of Medicine, University of Kelaniya, Ragama Sri Lanka, Phone: 447492063473, e-mail: pramodh@sltnet.lk
Population-based Cross-sectional study. World J Endoc Surg 2015;7(3):55-59.

Source of support: National Science Foundation. Grant no: 2006/04.

\section{Conflict of interest: None}

\section{BACKGROUND}

Epidemiology of goiters in Sri Lanka was formerly studied by Mahadeva and Shanmuganathan in 1967 who proposed an endemic goiter belt encompassing the wet zone and the parts of central hills (Fig. 1). ${ }^{1}$ Subsequent studies by Fernando MA et al reported a more widespread prevalence with pockets of high prevalence in the wet zone. ${ }^{2}$ Iodization of salt in Sri Lanka was implemented in 1995 as a universal strategy to prevent iodine deficiency. Study done in the year 2000 reported a shift in the distribution of endemicity to the north, central and eastern parts of the island in the post universal salt iodization era. ${ }^{3}$ These observations were made in studies done based on school children as a proxy sample. A rising level of antibodies among school children in the post iodization era was detected by Premawardhena et al suggesting the emergent of autoimmune thyroiditis as a causative factor for goiters other than iodine deficiency. ${ }^{4}$ The current study demonstrates a further change in the prevalence of goiters in Sri Lanka based on an island wide population based survey with Geographic information system (GIS) mapping of the prevalence pattern done for the first time. Geographic information system is a technique that helps to analyze spatio-temporal data recorded in relation to a geographic location and develop a map showing prevalence patterns. This technology is increasingly being used in public health-related data analysis which can demonstrate specific patterns of distribution of disease in relation to geographic variations. The aim of the study is to describe the actual prevalence of goiters in the Sri Lankan population and map the distribution with GIS.

\section{METHODS}

A descriptive cross-sectional study was carried out involving all areas of the country except for the northern province and Batticaloa district in the eastern province 
from August 2007 to June 2008. Ethical clearance was granted by the ethics review committee of the Faculty of Medicine of the University of Kelaniya, Sri Lanka. The country was divided into six geographical areas based on rain a fall pattern which is described in a previous publication by the authors. ${ }^{5}$ A multi-staged cluster sampling method was used. The required minimum sample size for each zone $(n=864)$ was calculated using a $10 \%$ estimated prevalence of goiter based on literature with an acceptable error of $2 \%$ on either side of the point estimate (total width of the $95 \%$ confidence interval-4\%). Based on logistical considerations it was decided to recruit a sample of 50 eligible individuals from 18 villages in each zone selected using the probability proportionate to size (PPS) method (Fig. 2). All individuals above 10 years of age were included in the study. The examination of study participants for goiter was done by at least two investigators and confirmed by the principal investigator. The goiters were graded according to the WHO grading. The goiter prevalence rates were visualized and interpolated using the Geographic Information System (ArcGIS 10.0, trial version). Cross-validation was done to ascertain a prediction error. This method sequentially omits a point in the dataset, predicting a value for that point's location using the rest of the data and compares the measured and predicted values. The model with a mean prediction error value closest to zero was used to identify the most appropriate goiter map to cover the entire country except the Northern Province.

\section{RESULTS}

The study included 108 'Grama Niladari' (GN) areas spanning across 88 Divisional Secretariats (DS). Grama Niladari divisions are administrative sub-districts divided based on population distribution. Out of 5200 individuals screened 426 goiters were detected. Sixty-six percent of the screened population was females (median age- -38 years, range 10-92). The calculated adjusted prevalence of goiters was $6.8 \%$. Highest goiter prevalence was reported from Kokmaduwa GN area (21.6\%) in the North Western province. The GN areas with more than $15 \%$ goiter prevalence were distributed across the country as pockets of high prevalence without any discernible pattern. Figure 3 shows the exact geographical locations of $\mathrm{GN}$ areas where the study was conducted. The prevalence levels in these locations were used to model and develop a map to

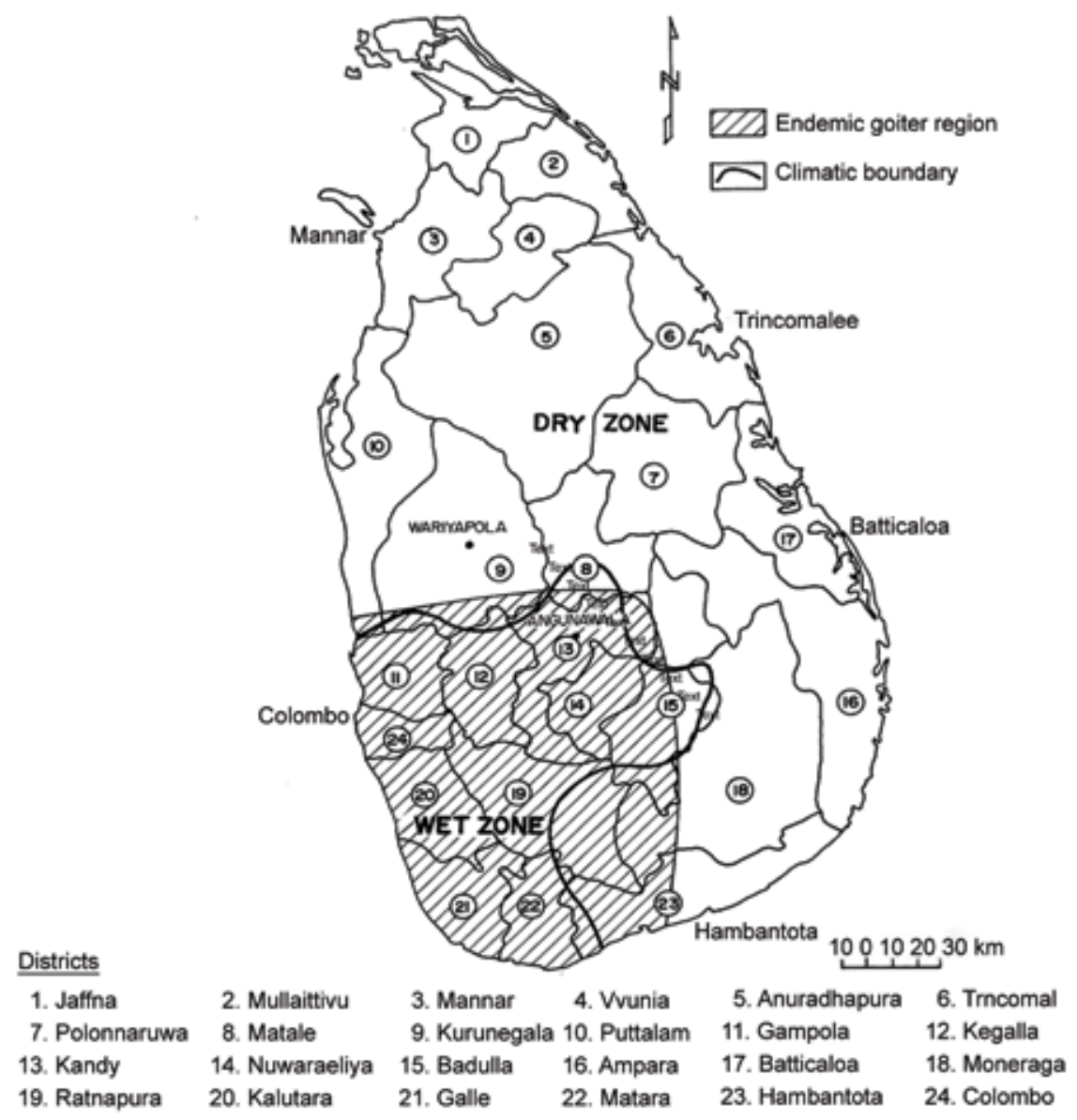

Fig. 1: Goiter belt in the wet zone of the country based on studies by Mahadevan and Shanmuganathan (1967) (Source: Dissanayake CB, Chandrajith R (1996) lodine in the environment and endemic goiter in Sri Lanka. In: Appleton JD, Fuge R, McCall GJH, editors) 


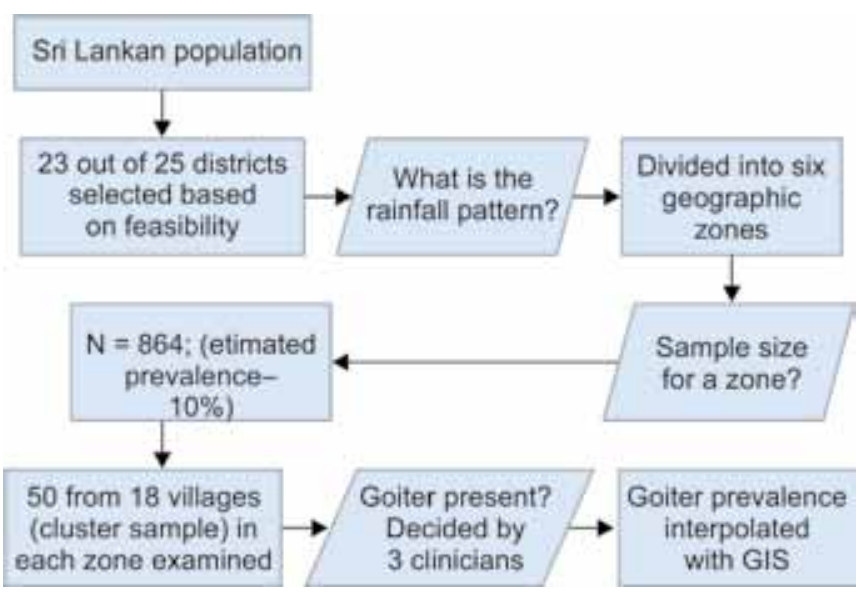

Fig. 2: Algorithm of the methodology used to assess the prevalence

show the goiter prevalence of entire country except the Northern Province. A series of models were developed using the inverse distance weighted (IDW) and Kriging interpolation methods with changing the input parameters to identify the best model to interpolate the goiter prevalence across the country. The mean prediction error closest to zero was resulted with a model developed by a Kriging method and was considered as the best interpolation method for the final goiter map of the country. Spatial data exploration using trend analysis and semivariogram cloud showed absence of a trend or autocorrelation in the collected data. Final interpolated map is shown in Figure 4.
The map indicates the clustering of geographic areas with similar goiter prevalence.

\section{DISCUSSION}

Earlier studies on goiter prevalence had shown a change in the prevalence patterns with time. ${ }^{2,3}$ The main limitation with the previous studies was that they were conducted on school children. The current study was a community based study excluding only the north and some districts in the east due to the security concerns which prevailed at the time of the study. The goiter prevalence rate observed during the current study was $6.8 \%$. Bangladesh and Pakistan, two neighboring countries from the south Asian region have reported goiter prevalence rates of $4.8 \%$ (2005) and 10.9\% (2014) respectively. ${ }^{6,7}$ Both studies were done on school children. Figures from India vary widely from $4.8 \%$ in the north west to $30.2 \%$ in the sub-himalayan region. 8,9 The concept of goiter belt in Sri Lanka was initially disputed by Fernando MA et al and Jayatissa $R$ et al who showed high degree of variability in the prevalence rates within the goiter belt. ${ }^{2,3}$ The maps developed by the previous occasions based on limited research findings and hypothesis differs from the goiter prevalence map generated in the present study. ${ }^{1,3}$ One of the strong suggestions of goiter distribution in Sri Lanka was existence of a 'goiter belt'. However, the present

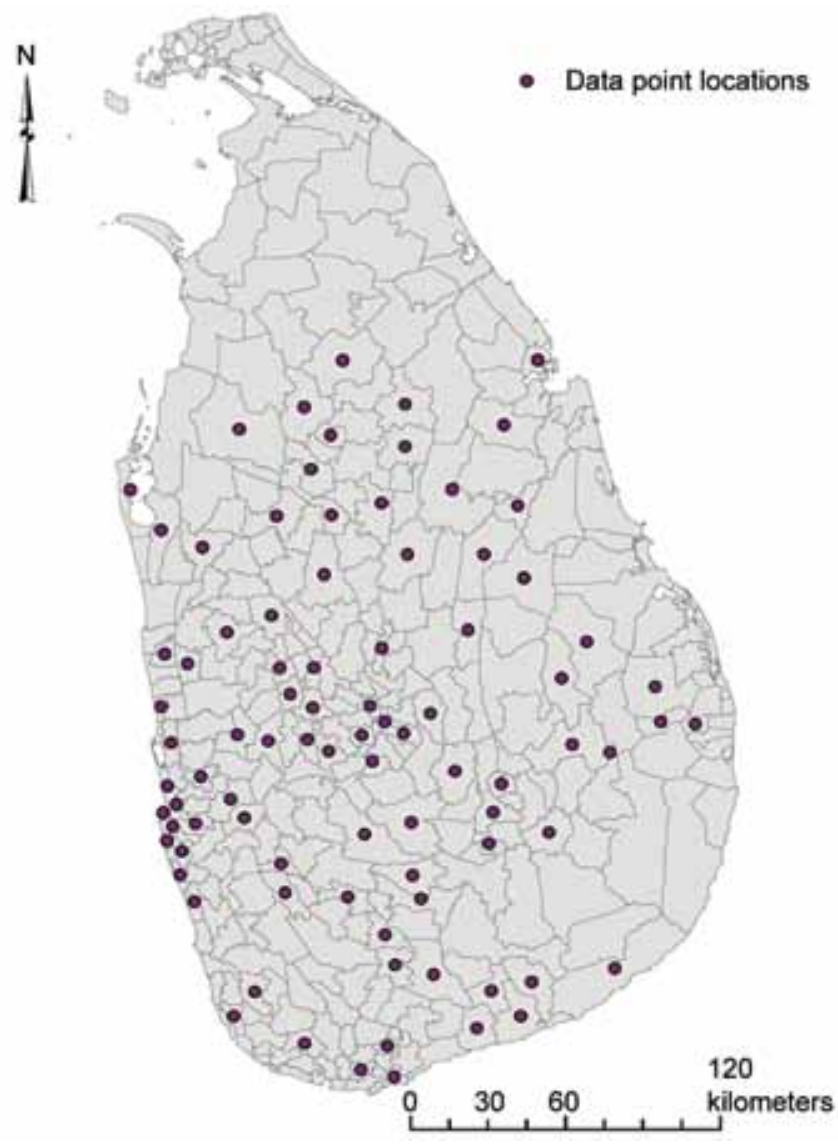

Fig. 3: Reference points of data collection used for interpolation 


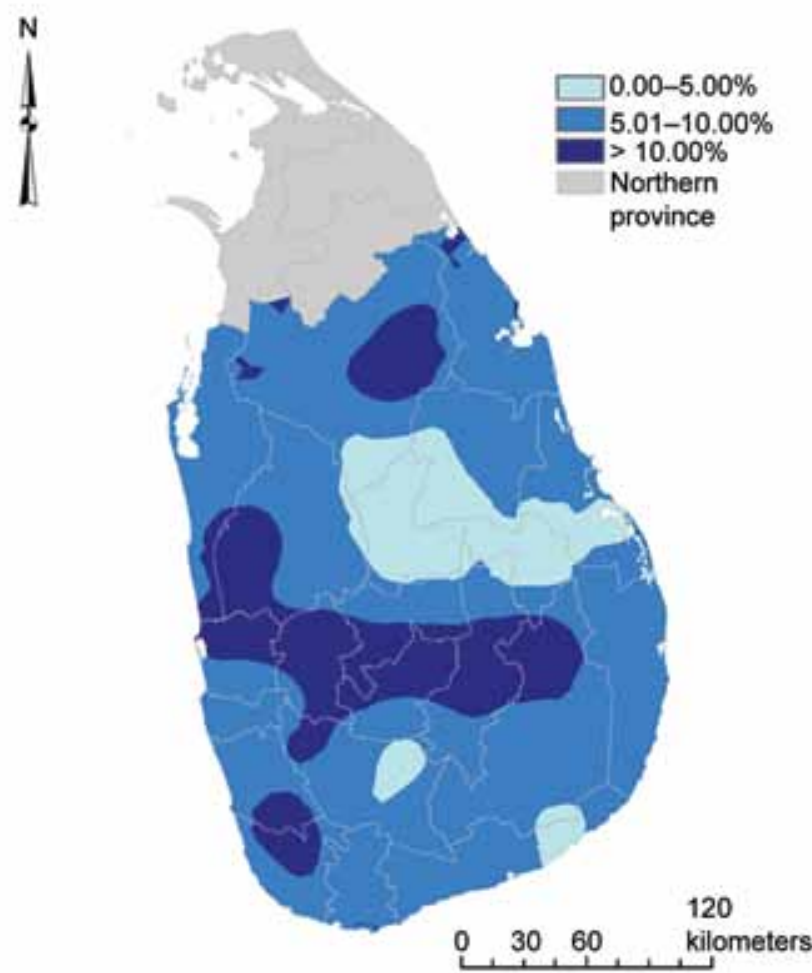

Fig. 4: Proposed epidemiological map with the distribution of goiter prevalence in Sri Lanka created using ArcGIS 10.0 software

study does not support for this phenomenon. Further, in contrast to the suggestions made by previous researchers, the rainfall pattern or geographical elevations in Sri Lanka do not overlay with the pattern of goiter prevalence identified in the present study. The map developed from the data of this study (Fig. 3) shows clustering of high prevalence areas in the western and central provinces and pockets of high prevalence distributed without a set pattern. The causal relationship between goiter and iodine deficiency may be changing in Sri Lanka. Previous researchers have observed rising levels of antibodies among school children comparatively in the preand post-global salt iodization. ${ }^{4}$ Based on other findings of the current study, the authors have reported a positive correlation between the urine iodine concentration and the presence of anti-thyroperoxidase antibodies in serum in a previous publication. ${ }^{4}$ Higher urinary iodine excretion than the expected average levels was reported from all studies geographical regions of the country. Based on these findings it was concluded that high iodine concentration in the consumed salt may be giving rise to a new pattern of goiters due to autoimmune thyroiditis. To our knowledge there is no any published disease prevalence visualization in Sri Lanka with interpolation of data for the non-studied areas. The current map would be the first digital representation of prevalence of a health condition in the country based on national representative data from the field. Geostatistical frameworks coupled with analysis have proven to provide robust data for decision making eliminating biases due to missing values. ${ }^{10,11}$ The GIS data visualization coupled with spatial analytical techniques helps to understand the spatial structure of a process. Prior to the selection of final model to develop the map, a vigorous literature search was done and many models were run with different interpolation techniques and input parameters using the latest software version of the Arch GIS. Thus, we expect that the proposed map gives the scientifically robust goiter prevalence distribution in Sri Lanka. This information would be helpful in the policy making process and resource allocation in the health sector.

\section{CONCLUSION}

The prevalence rate of goiter in the community is $6.8 \%$. Island wide distribution of the prevalence is different to prior research findings and the hypothesis pertaining to the goiter prevalence pattern in Sri Lanka. There is a wide spread prevalence with clustering in some regions and scattered prevalence throughout the country. Concept of an endemic goiter belt based on rainfall and elevation seems to be no longer applicable. Integration of GIS has helped to develop a comprehensive epidemiological map of the disease burden.

\section{ACKNOWLEDGMENT}

Ranil Fernando, Pramodh Chandrasinghe were involved in data collection, data analysis, writing manuscript and 
critical appraisal of the paper. Sumal Nandasena, Arunasalam Pathmeswaran were involved in data analysis, writing manuscript and critical appraisal of the paper.

We thank all participants for consenting for the study. Authors wish to thank the National Science Foundation of Sri Lanka for funding the field data collection component of this study.

\section{REFERENCES}

1. Mahadeva K, Senthe Shanmuganathan S. The problem of goiter in Ceylon. Br J Nutrition 1967;21:341-352.

2. Fernando MA, Balasuriya S, Herath KB, Katugampola S. Endemic iodization in Sri Lanka. Asia-Pacific J Public Health 1989;3:11-19.

3. Jayatissa R, Gunathilaka MM. Iodine Nutrition Status in Sri Lanka 2005. Department of Nutrition Medical Research Institute Department of Healthcare and Nutrition 2006.

4. Premawardhana LDKE, Parker PPA, Wijeyaratne CN, Jayasingha A, De Silva DGH, Lazarm JH. Increased prevalence of thyroglobulin antibodies in Sri Lankan schoolgirls-is iodine the cause? Eur J Endocrinol 2000;143: 185-188.

5. Fernando R, Chandrasinghe P, Pathmeswaran A. The prevalence of autoimmune thyroiditis after universal salt iodisation in Sri Lanka. Ceylon Med J 2012 Sep;57(3):116-119.
6. Yusuf HK, Rahman AM, Chowdhury FP, Mohiduzzaman M, Banu CP, Sattar MA, Islam MN. Iodine deficiency disorders in Bangladesh, 2004-05: ten years of iodized salt intervention brings remarkable achievement in lowering goiter and iodine deficiency among children and women. Asia Pacific J Clin Nutrition 2008;17(4):620-628.

7. Saira S, Khattak RM, Rehman AU, Khan AA, Khattak MNK. Prevalence of goiter and iodine status in 6-12 years school children and pregnant women of district Charsadda, Pakistan. ActaEndocrinologica (Buc); 10;1:65-75.

8. Makwana NR, Shah VR, Unadkat S, Shah HD, Yadav S. Goiter prevalence and current iodine deficiency status among school age children years after the universal salt iodization in Jamnagar district, India. Thyroid Research and Practice 2012;9:2:40-44.

9. Chandra AK, Bhattacharjee A, Malik T, Ghosh S. Goiter prevalence and iodine nutritional status of school children in a sub-himalayan tarai region of eastern Uttar Pradesh. Indian Paediatrics 2008;45(6):454-455.

10. Gething PW, Noor AM, Goodman CA, Gikandi PW, Hay SI, Sharif SK, Atkinson PM, Snow RW. Information for decision making from imperfect national data: tracking major changes in health care use in Kenya using geostatistics. BMC Medicine 2007 Dec 11;5:37.

11. Moore DA, Carpenter TE. Spatial analytical methods and geographic information systems: use in health research and epidemiology. Epidemiologic Reviews 1997;21(2):143-161. 